\title{
Self-excited oscillations of charge-spin accumulation due to single-electron tunneling
}

\author{
D. Radić, ${ }^{1,2,3}$ A. M. Kadigrobov, ${ }^{2,4}$ L. Y. Gorelik, ${ }^{1}$ R. I. Shekhter, ${ }^{2}$ and M. Jonson ${ }^{2,5,6}$ \\ ${ }^{1}$ Department of Applied Physics, Chalmers University of Technology, SE-412 96 Göteborg, Sweden \\ ${ }^{2}$ Department of Physics, University of Gothenburg, SE-412 96 Göteborg, Sweden \\ ${ }^{3}$ Department of Physics, Faculty of Science, University of Zagreb, 1001 Zagreb, Croatia \\ ${ }^{4}$ Theoretische Physik III, Ruhr-Universität Bochum, D-44801 Bochum, Germany \\ ${ }^{5}$ School of Engineering and Physical Sciences, Heriot-Watt University, Edinburgh EH14 4AS, Scotland, United Kingdom \\ ${ }^{6}$ Division of Quantum Phases and Devices, School of Physics, Konkuk University, Seoul 143-701, Korea
}

(Received 2 June 2010; published 9 September 2010)

\begin{abstract}
We theoretically study electronic transport through a layer of quantum dots connecting two metallic leads. By the inclusion of an inductor in series with the junction, we show that steady electronic transport in such a system may be unstable with respect to temporal oscillations caused by an interplay between the Coulomb blockade of tunneling and spin accumulation in the dots. When this instability occurs, a new stable regime is reached, where the average spin and charge in the dots oscillate periodically in time. The frequency of these oscillations is typically on the order of $1 \mathrm{GHz}$ for realistic values of the junction parameters.
\end{abstract}

DOI: 10.1103/PhysRevB.82.125311

PACS number(s): 73.23.Hk, 73.63.Kv

\section{INTRODUCTION}

During the last decade, spin-polarized electronic transport through quantum dots (QDs) connecting two metallic ferromagnetic $(\mathrm{F})$ leads has been the subject of very intensive both theoretical and experimental research with numerous applications in spin-based devices such as spin valves, spin filters, spin diodes, etc. ${ }^{1-3}$ Ferromagnetic ordering in the leads, which causes the rates for tunneling on-to and outfrom the dot to depend on the electron spin, results in the accumulation of spin in the dots ${ }^{4,5}$ in addition to charge. This spin accumulation affects the transport properties of the system $^{6,7}$ and provides a way to control the spin polarization of the current by bias or gate voltages. Moreover, it was found that the Coulomb blockade phenomenon significantly affects the dc (Refs. 8-11) and the shot noise ${ }^{12}$ in such systems, which has provided new opportunities for their electrical manipulation. ${ }^{13}$

In most previous papers, time-independent effects caused by the spin and charge accumulations on the dots between ferromagnetic leads have been investigated. In this paper we focus on self-induced time-dependent phenomena which may arise in such systems under a static bias with an inductor included in series with the junction. We will show that under certain conditions, time-independent electronic transport across a layer of quantum dots placed between normal $(\mathrm{N})$ and magnetic leads becomes unstable, which results in oscillations in time of spin and charge accumulations in the layer of dots. One may assign this instability to the existence of a negative differential conductance (NDC), arising due to an interplay between the Coulomb blockade and the spin blockade phenomena. The occurrence of an NDC in systems similar to the one considered by us was reported in Refs. 14-18. However, we will show that in our case, an instability of the time-independent regime of the charge and spin flow may arise even at a positive differential conductance of the junction.

\section{MODEL}

We consider a layer of identical quantum dots, with two spin-dependent states, connecting $\mathrm{N}$ and $\mathrm{F}$ metallic leads. All dots in the layer are supposed to be placed at the same distance with respect to the leads (see Fig. 1). The system under consideration is described by the Hamiltonian

$$
H=\sum_{i=\mathrm{N}, \mathrm{F}} H_{i}^{\text {lead }}+H^{\mathrm{QD}}+\sum_{i=\mathrm{N}, \mathrm{F}} H_{i}^{\text {tunnel }},
$$

where the partial Hamiltonians

$$
\begin{gathered}
H_{i}^{\text {lead }}=\sum_{\vec{k}, \sigma} E_{\vec{k}, \sigma, i} a_{\vec{k}, \sigma, i}^{\dagger} a_{\vec{k}, \sigma, i}, \\
H^{\mathrm{QD}}=\sum_{\sigma, n} \epsilon_{\sigma} c_{\sigma, n}^{\dagger} c_{\sigma, n}+U c_{\uparrow, n}^{\dagger} c_{\uparrow, n} c_{\downarrow, n}^{\dagger} c_{\downarrow, n}, \\
H_{i}^{\text {tunnel }}=\tau_{i} \sum_{\vec{k}, \sigma}\left[a_{\vec{k}, \sigma, i}^{\dagger} c_{\sigma, n}+\text { H.c. }\right]
\end{gathered}
$$

describe electrons in the leads, in the QDs, and tunneling coupling between QDs and leads, respectively. Here $a_{\vec{k}, \sigma, i}^{\dagger}$ creates an electron with wave vector $\vec{k}$ and spin $\sigma=\uparrow, \downarrow$ in the corresponding lead $i=\mathrm{N}, \mathrm{F}$ ( $i$ is the lead index); $E_{\vec{k}, \sigma, \mathrm{N}}$ $=\varepsilon(k)$ and $E_{\vec{k}, \sigma, \mathrm{F}}=\varepsilon(k)-I_{\sigma}$, where $\varepsilon(k)$ is electron kinetic energy and $I_{\downarrow}=-I_{\uparrow} \equiv I$ is the ferromagnetic exchange energy; $c_{\sigma, n}^{\dagger}$ creates an electron with spin $\sigma$ and energy $\epsilon_{\sigma}$ in the $n$th

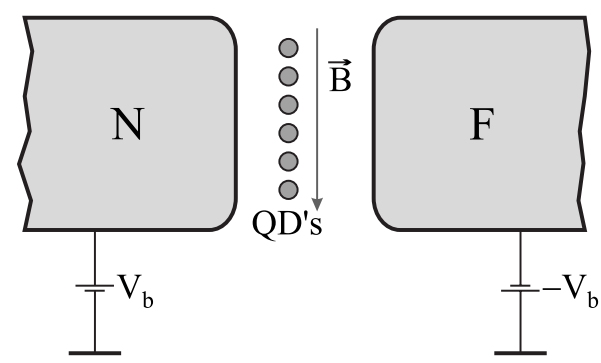

FIG. 1. Charge and spin transport through a layer of quantum dots sandwiched between a $\mathrm{N}$ and a $\mathrm{F}$ lead subjected to an external magnetic field $\vec{B}$ is studied. The junction is symmetrically biased by the voltage $2 V_{b}$. 


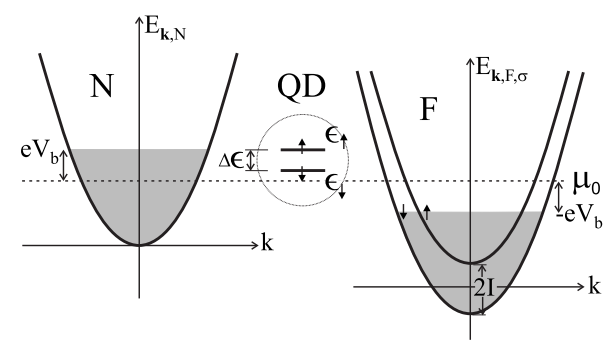

FIG. 2. Electronic energy scheme (P configuration) showing the nonmagnetic band (N) and the bands for $\uparrow$ (minority) and $\downarrow$ (majority) spins in the F. The latter are split by $2 I$, where $I$ is the ferromagnetic exchange energy. The applied bias voltage $2 V_{b}$ shifts the electron energy by $\pm e V_{b}$ in $\mathrm{N} / \mathrm{F}$ lead, respectively, from the chemical potential $\mu_{0}$. Here $\epsilon_{\downarrow}$ and $\epsilon_{\uparrow}$ are the spin-dependent energy levels in the dots.

dot $(n=1,2, \ldots, \mathcal{N})$ counted from the identical chemical potentials of electrons in the metallic leads $\mu_{0}$; $U$ is the Coulomb interaction energy due to the double occupancy of the QD level by electrons with opposite spins. We consider the case when the interdot distance is much larger than the distance between the metallic leads allowing us to neglect the interdot Coulomb interactions. We set $\mu_{0}$ as an origin for measuring all energies. The difference between spin "up" and "down" energy levels $\Delta \epsilon=\epsilon_{\uparrow}-\epsilon_{\downarrow}$ in QD can be controlled, e.g., by the Zeeman splitting induced by the applied external magnetic field $\vec{B}$ which we will fix, for the sake of definiteness, in down direction $\left[\Delta \epsilon \approx 10^{-2}-10^{-1} \mathrm{meV}\right.$ in the magnetic field 0.1-1 T (Ref. 19)]. The external magnetic field and the F-lead magnetization are taken parallel in this model, so no spin precession effects, as investigated, e.g., in Ref. 20, are present. We will consider both the possible orientations of F-lead magnetization: along the magnetic field direction (P) and opposite to it (A). Furthermore, due to the exponential sensitivity of the tunneling matrix elements $\tau_{i}$ to the geometrical position of the QD with respect to the leads, physically interesting limiting cases can be achieved. We have studied two such cases: the $\mathrm{N}$ junction, in which the QDs are closer to the $\mathrm{N}$ lead $\left(\tau_{\mathrm{N}} \gg \tau_{\mathrm{F}}\right)$, and the $\mathrm{F}$ junction where they are closer to the F lead $\left(\tau_{\mathrm{F}} \gg \tau_{\mathrm{N}}\right)$.

To study charge and spin transfer between leads we will consider, for the sake of simplicity, a symmetric voltage biasing of the junction (see Fig. 1). In this case the bias voltage does not affect the position of the QD levels $\epsilon_{\sigma}$ but it shifts the chemical potentials in the leads (see Fig. 2). In this paper we will restrict our study to the case when $\epsilon_{\downarrow}>0$. The magnitude of the splitting $\Delta \epsilon=\epsilon_{\uparrow}-\epsilon_{\downarrow}$ is assumed to be substantially larger than the intrinsic level width and $k_{B} T \ll \Delta \epsilon$ ( $T$ is the temperature) thus providing well-controlled separation of the spin states. Furthermore we will consider Coulomb blockade regime when temperature and bias voltage are much less then the double charging energy $U$. The conditions above determine the lack of charge and spin transfer if the absolute value of bias voltage $\left|V_{b}\right|$ is less then $V_{\sigma}=\left|\epsilon_{\sigma} / e\right|$ (e is the electron charge). When $\left|V_{b}\right|$ exceeds $V_{l}$, the $\downarrow$-spin polarized tunneling arise manifesting itself as a step in the current-voltage characteristic (IVC) at $\left|V_{b}\right|=V_{\downarrow}$. By further increase in the bias voltage an additional step appears at
$\left|V_{b}\right|=V_{\uparrow}$ when $\uparrow$-spin polarized tunneling is triggered. However, due to the Coulomb blockade, the presence of an electron in one state effectively blocks the current through the other one, thus affecting the IVC. We will show that a negative differential conductance can be achieved in the vicinity of $V_{\uparrow}$ if the tunneling rate of the electron transfer between the $\uparrow$ state in the dot and the drain electrode is low enough compared to the one for the $\downarrow$ state (spin blockade). ${ }^{17}$

To analyze quantitatively kinetic properties of the system under consideration we will use rate equations for the probabilities $P_{\uparrow(\downarrow)}$ to find electron with spin $\uparrow(\downarrow)$ on the dot. They can be derived from the generalized master equation for the density matrix by adopting the Markovian approximation in the limit of weak tunneling (see the Appendix). ${ }^{19,21}$ Double occupation of the dots is prohibited by the Coulomb blockade thus leaving only two independent components in the rate equations (the probability to find the dot unoccupied is $\left.P_{0}=1-P_{\uparrow}-P_{\downarrow}\right)$. The combinations $P_{c}=P_{\uparrow}+P_{\downarrow}$ and $P_{s}=P_{\uparrow}$ $-P_{\downarrow}$ then describe charge and spin accumulations in the layer, respectively. The rate equations describing time evolution of the average dot populations can be presented in the form

$$
\frac{1}{\Gamma_{\mathrm{N}}} \frac{d P_{\sigma}}{d t}=-\left(1+\gamma_{\sigma}\right) P_{\sigma}+\left[f_{\mathrm{N}}^{\sigma}(V)+f_{\mathrm{F}}^{\sigma} \gamma_{\sigma}(V)\right]\left(1-P_{-\sigma}\right) .
$$

Here $\Gamma_{\mathrm{N}}=2 \pi\left|\tau_{\mathrm{N}}\right|^{2} g_{\mathrm{N}}, g_{\mathrm{N}}$ is the density of electronic states in the normal lead, which we assume to be energy independent,

$$
\gamma_{\sigma} \equiv \frac{\Gamma_{\mathrm{F}}^{\sigma}}{\Gamma_{\mathrm{N}}}=\left(\frac{\tau_{\mathrm{F}}}{\tau_{\mathrm{N}}}\right)^{2} \frac{g_{\mathrm{F}}^{\sigma}}{g_{\mathrm{N}}}
$$

where $g_{\mathrm{F}}^{\sigma}$ is the spin-dependent density of electronic states in the ferromagnetic lead,

$$
\begin{gathered}
f_{\mathrm{N} \sigma}(V)=f_{\mathrm{F} \sigma}(-V) \equiv f\left(\epsilon_{\sigma}-e V\right), \\
f(\varepsilon)=\frac{1}{1+e^{\varepsilon / k_{B} T}},
\end{gathered}
$$

where $2 V$ is the total voltage drop across the junction.

The average current per one dot through the $\mathrm{N} \rightarrow \mathrm{QD}$ junction is given by the expression

$$
j(V)=e \Gamma_{\mathrm{N}} \sum_{\sigma}\left[-P_{\sigma}+f_{\mathrm{N} \sigma}(V)\left(1-P_{-\sigma}\right)\right] .
$$

The geometrical asymmetry parameter $\xi \equiv\left|\tau_{\mathrm{F}} / \tau_{\mathrm{N}}\right|^{2}$ classifies two types of junctions: $\mathrm{N}$ junction for $\xi \ll 1$ and $\mathrm{F}$ junction for $\xi \gg 1$.

\section{III. dc TRANSPORT}

Solving the system [Eq. (3)] at $V=V_{b}=$ const we obtain the time-independent occupation probabilities 


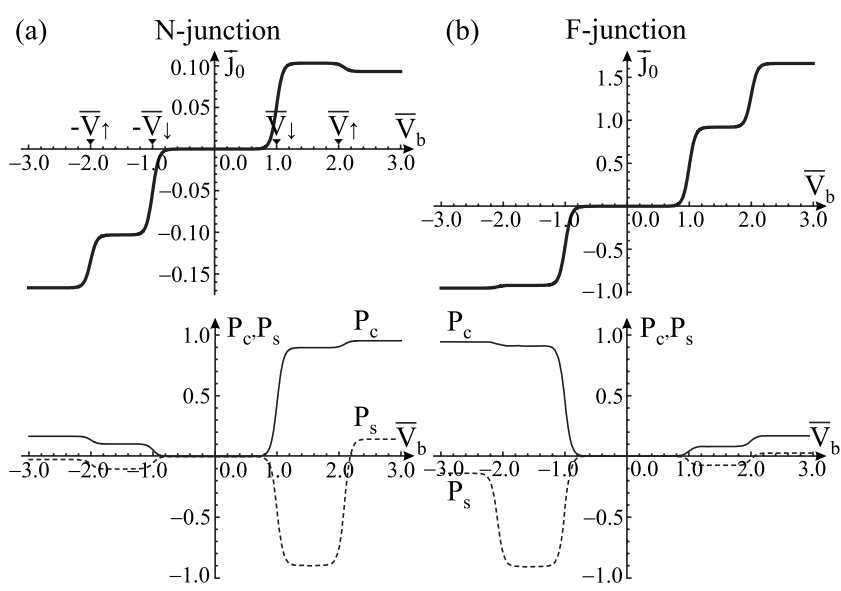

FIG. 3. Single-dot IVC with corresponding on-dot charge $\left(P_{c}\right)$ and spin $\left(P_{s}\right)$ accumulation probabilities for: (a) an N-type junction with $\gamma_{\uparrow}=0.085$ and $\gamma_{\downarrow}=0.115(\xi=0.1)$ and (b) an F-type junction with $\gamma_{\uparrow}=8.5$ and $\gamma_{\downarrow}=11.5(\xi=10)$. The current is normalized as $\overline{j_{0}}=j_{0} / e \Gamma_{\mathrm{N}}$. We take $\epsilon_{\uparrow}=2 \epsilon_{\downarrow}$, temperature $k_{B} T / \Delta \epsilon=0.05$ and introduce the dimensionless voltages $\bar{V}=e V / \Delta \epsilon ; \bar{V}_{\sigma}=\epsilon_{\sigma} / \Delta \epsilon$. One can see that the IVC is not symmetric with respect to voltage biasing $\overline{V_{b}} \rightarrow-\overline{V_{b}}$. Also, in NF biasing, the N-type junction produces a NDC around $\bar{V}_{\uparrow}=2$ in contrast to the F-type junction. For FN biasing the NDC is absent in both cases.

$$
P_{\sigma}^{s t}\left(V_{b}\right)=\frac{\left(1+\gamma_{-\sigma}\right)\left(f_{\mathrm{N} \sigma}+f_{\mathrm{F} \sigma} \gamma_{\sigma}\right)-\prod_{\sigma^{\prime}}\left(f_{\mathrm{N} \sigma^{\prime}}+f_{\mathrm{F} \sigma^{\prime}} \gamma_{\sigma^{\prime}}\right)}{\prod_{\sigma^{\prime}}\left(1+\gamma_{\sigma^{\prime}}\right)-\prod_{\sigma^{\prime}}\left(f_{\mathrm{N} \sigma^{\prime}}+f_{\mathrm{F} \sigma^{\prime}} \gamma_{\sigma^{\prime}}\right)} .
$$

Inserting expression (7) into (6), one gets the dc per dot, $j_{0}\left(V_{b}\right)$, through the junction. The IVC together with corresponding charge and spin accumulation probabilities are presented in Fig. 3.

From expression (6) and Fig. 3 it is evident that the IVC is not symmetric with respect to a change in sign of the bias voltage. In our further investigation we will focus our attention on the phenomena occurring by activation of the spin-up channel of the electron transfer. Therefore, we will consider the bias voltage values $\left|V_{b}\right| \approx V_{\uparrow}$. Taking into account the fact that $\left|e V_{b}\right|-\epsilon_{\downarrow} \approx \Delta \epsilon \gg k_{B} T$, one can distinguish two cases: (a) $\mathrm{NF}$ biasing $\left[e V_{b}>0 ; f_{\mathrm{F} \sigma}\left(V_{b}\right) \approx 0, f_{\mathrm{N} \downarrow}\left(V_{b}\right) \approx 1\right]$ and $(\mathrm{b}) \mathrm{FN}$ biasing $\left[e V_{b}<0 ; f_{\mathrm{N} \sigma}\left(V_{b}\right) \approx 0, f_{\mathrm{F} \downarrow}\left(V_{b}\right) \approx 1\right]$ in which we obtain an approximate expression for dc, accurate up to the exponentially small correction $\exp \left(-\Delta \epsilon / k_{B} T\right) \ll 1$.

(a) NF biasing

$$
j_{0}\left(V_{b}\right) \approx e \Gamma_{\mathrm{N}} \gamma_{\downarrow} \frac{1+\gamma_{\uparrow}-f_{\mathrm{N} \uparrow}\left(1-\gamma_{\uparrow}\right)}{\left(1+\gamma_{\uparrow}\right)\left(1+\gamma_{\downarrow}\right)-f_{\mathrm{N} \uparrow}} .
$$

From Eq. (8) it is clearly seen that the voltage dependence of the stationary IVC is entirely driven by the Fermi function $f_{\mathrm{N} \uparrow}\left(V_{b}\right)$ which exhibits a step-uplike behavior at $\left|V_{b}\right|=V_{\uparrow}$. Differentiating Eq. (8) with respect to voltage we find the differential conductance
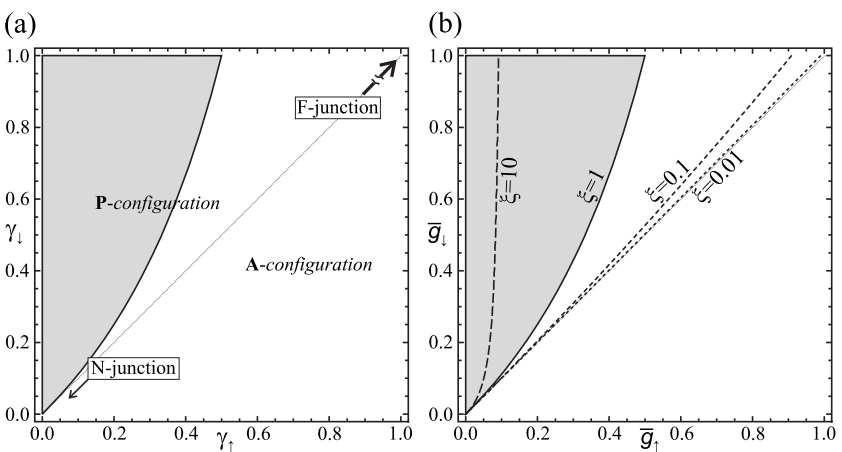

FIG. 4. (a) An IVC section with a NDC around $V_{b}=V_{\uparrow}$ appears for parameters $\gamma_{\uparrow}$ and $\gamma_{\downarrow}$ within the range corresponding to the shaded area based on the condition $\vartheta\left(\gamma_{\uparrow}, \gamma_{\downarrow}\right)<0$. (b) The same condition expressed in terms of the normalized densities of states $\bar{g}_{\sigma} \equiv g_{\mathrm{F}}^{\sigma} / g_{\mathrm{N}}$ for various values of the geometrical asymmetry parameter $\xi$ characterizing $\mathrm{N}$ junctions $(\xi<1)$ and $\mathrm{F}$ junctions $(\xi>1)$. The diagonal line divides the region of parameters for the $\mathbf{P}$ configuration $\left(\bar{g}_{\downarrow}>\bar{g}_{\uparrow}\right)$ and the A configuration $\left(\bar{g}_{\downarrow}<\bar{g}_{\uparrow}\right)$. Evidently, NDC exists in $\mathbf{P}$ configuration only.

$$
\frac{d j_{0}}{d V_{b}}=-e^{2} \Gamma_{\mathrm{N}} f_{\mathrm{N} \uparrow}^{\prime} \frac{\gamma_{\downarrow}\left(1+\gamma_{\uparrow}\right)}{\left[\left(1+\gamma_{\uparrow}\right)\left(1+\gamma_{\downarrow}\right)-f_{\mathrm{N} \uparrow}\right]^{2}} \vartheta\left(\gamma_{\uparrow}, \gamma_{\downarrow}\right),
$$

where

$$
\begin{gathered}
\vartheta\left(\gamma_{\uparrow}, \gamma_{\downarrow}\right) \equiv \gamma_{\uparrow}-\gamma_{\downarrow}+\gamma_{\uparrow} \gamma_{\downarrow}, \\
\left.f_{\mathrm{N} \uparrow}^{\prime} \equiv \frac{d f}{d \varepsilon}\right|_{\epsilon_{\uparrow}-e V_{b}}<0 .
\end{gathered}
$$

The corresponding current "jump" $\Delta j_{0} \equiv j_{0}\left(-V_{\uparrow}+\delta V\right)$ $-j_{0}\left(-V_{\uparrow}-\delta V\right)$, where $\Delta \epsilon \gg|e \delta V| \gg k_{B} T$, is

$$
\Delta j_{0}=e \Gamma_{\mathrm{N}} \frac{\gamma_{\downarrow}}{\left(1+\gamma_{\downarrow}\right)\left(\gamma_{\uparrow}+\gamma_{\downarrow}+\gamma_{\uparrow} \gamma_{\downarrow}\right)} \vartheta\left(\gamma_{\uparrow}, \gamma_{\downarrow}\right)
$$

in which $\vartheta\left(\gamma_{\uparrow}, \gamma_{\downarrow}\right)$ determines the sign of the jump.

From Eq. (9) it can be seen that the IVC exhibits NDC, $d j_{0} / d V_{b}<0$, if $\gamma_{\uparrow}$ and $\gamma_{\downarrow}$ satisfy condition $\vartheta\left(\gamma_{\uparrow}, \gamma_{\downarrow}\right)<0$ shown graphically in Fig. 4. To lowest order in the small parameter formed by the ratio of the polarization parameter and the Fermi energy, $I / \epsilon_{\mathrm{F}} \ll 1$, this inequality may be written as

$$
\frac{g_{\mathrm{F}}^{\downarrow}-g_{\mathrm{F}}^{\uparrow}}{g_{\mathrm{N}}} \sim \frac{I}{\epsilon_{\mathrm{F}}}>\xi .
$$

Therefore, the smaller is $\xi$, the smaller ferromagnetic polarization of F lead is required for NDC. Obviously, NDC is present only in $\mathbf{P}$ configuration. As it is seen from Eqs. (5) and (14) the differential conductance [Eq. (9)] is proportional to $T^{-1}$ at $V_{b}=-V_{\uparrow}$. Since it exponentially decreases at $\mid V_{b}$ $+V_{\uparrow}\left|\gg k_{B} T /\right| e \mid$, the width of the jump in IVC is $\sim k_{B} T$.

(b) FN biasing

$$
j_{0}\left(V_{b}\right) \approx-e \Gamma_{\mathrm{N}} \frac{f_{\mathrm{F} \uparrow} \gamma_{\uparrow}\left(1-\gamma_{\downarrow}\right)+\gamma_{\downarrow}\left(1+\gamma_{\uparrow}\right)}{\left(1+\gamma_{\uparrow}\right)\left(1+\gamma_{\downarrow}\right)-f_{\mathrm{F} \uparrow} \gamma_{\uparrow} \gamma_{\downarrow}} .
$$

The differential conductance is 


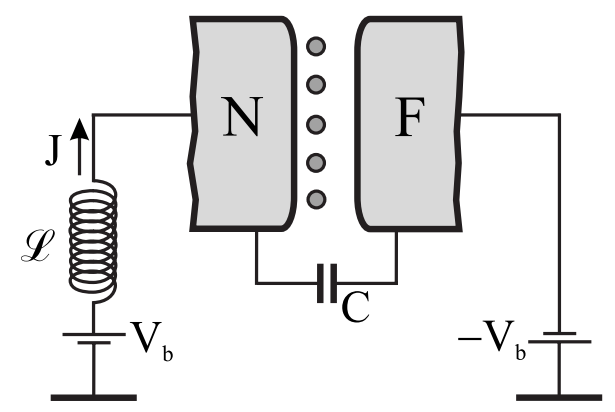

FIG. 5. Circuit scheme used for the instability investigation: $C$ is the intrinsic junction capacitance, $\mathcal{L}$ is the inductance of the circuit, and $J$ is the current flowing through the inductor.

$$
\frac{d j_{0}}{d V_{b}}=-e^{2} \Gamma_{\mathrm{N}} f_{\mathrm{F} \uparrow}^{\prime} \frac{\gamma_{\uparrow}\left(1+\gamma_{\uparrow}\right)}{\left[\left(1+\gamma_{\uparrow}\right)\left(1+\gamma_{\downarrow}\right)-f_{\mathrm{F} \uparrow} \gamma_{\uparrow} \gamma_{\downarrow}\right]^{2}},
$$

where

$$
\left.f_{\mathrm{F} \uparrow}^{\prime} \equiv \frac{d f}{d \varepsilon}\right|_{\varepsilon_{\uparrow}+e V_{b}}<0 .
$$

One can see that NDC does not appear in the FN biasing of the junction.

The asymmetry of IVC with respect to the direction of bias voltage for junctions with magnetic leads is known and utilized, e.g., for spin filtering, spin diodes, etc. ${ }^{2,3,18}$ In the system under consideration NDC also occurs only at the regime of an NF biasing. Physics of this phenomenon, observed experimentally, ${ }^{15}$ is most transparent in the case of a strongly asymmetric N-P junction $\left(\gamma_{\uparrow} \ll \gamma_{\downarrow} \ll 1\right)$ at low temperatures. Indeed, under such conditions and at $\epsilon_{\downarrow}<e V_{b}$ $<\epsilon_{\uparrow}$ spin up states in the dots are not populated $\left(P_{\uparrow}=0\right)$ while those for spin down are almost completely populated $\left(P_{\downarrow} \simeq 1\right)$, giving the average current $j_{0} \simeq e \Gamma_{\mathrm{N}} \gamma_{\downarrow}$. Then at $e V_{b}>\epsilon_{\uparrow}$ the up-spin states start to contribute and the electrons are mostly trapped in these states $\left(P_{\uparrow} \simeq 1\right)$ due to a very long escape time $\simeq\left(\gamma_{\uparrow} \Gamma_{\mathrm{N}}\right)^{-1}$ that blocks the current through the spin-down states $\left(P_{\downarrow} \simeq \gamma_{\uparrow} / \gamma_{\downarrow} \ll 1\right)$ by the Coulomb blockade effect. Consequently, for $e V_{b}>\epsilon_{\uparrow}$ the average current $j_{0} \simeq e \Gamma_{\mathrm{N}}\left(\gamma_{\uparrow} P_{\uparrow}+\gamma_{\downarrow} P_{\downarrow}\right) \simeq 2 e \Gamma_{\mathrm{N}} \gamma_{\uparrow}$ is less than the average current at $e V_{b}<\epsilon_{\uparrow}$.

\section{DYNAMICAL INSTABILITY OF CHARGE-SPIN ACCUMULATIONS}

In this section we will only consider an $\mathrm{N}$ junction $\left(\gamma_{\sigma}\right.$ $\leq 1)$ in the regime of NF biasing which is the most interesting one. Really, a voltage biased circuit with a nonlinear resistor, $R(V)$, providing the section with NDC in the IVC, is usually considered as a prerequisite for dynamical instability of the system. To investigate stability of charge/spin accumulation in our system we introduce an inductor with inductance $\mathcal{L}$ in series with the junction (see Fig. 5). We also take into account the capacitance of the junction $C \approx \mathcal{N} C_{1 \mathrm{QD}}$, where $\mathcal{N}$ is the number of quantum dots inside the junction, $C_{1 \mathrm{QD}}=\varepsilon l^{2} / d$ is the average capacitance per one dot $(\varepsilon$ is the dielectric constant of the layer material, $l$ is the average distance between dots, and $d$ is the distance between the leads).
The tunneling and electrical processes in this system are governed by the system of four differential equations,

$$
\begin{gathered}
\frac{1}{\Gamma_{\mathrm{N}}} \frac{d P_{\sigma}}{d t}+\left(1+\gamma_{\sigma}\right) P_{\sigma}-f_{\mathrm{N} \sigma}(V)\left(1-P_{-\sigma}\right)=0, \\
\mathcal{L} \frac{d J}{d t}+2 V=2 V_{b}, \\
\mathcal{N} j\left(V, P_{\sigma}\right)+2 C \frac{d V}{d t}=J .
\end{gathered}
$$

Here $J$ is the total current flowing through the inductor and the current per one dot $j\left(V, P_{\sigma}\right)$ is given by Eq. (6). The set of differential equation [Eq. (16)] always has the timeindependent solution

$$
P_{\sigma}=P_{\sigma}^{s t}, \quad V=V_{b}, \quad J=\mathcal{N} j\left(V_{b}, P_{\sigma}^{s t}\right)
$$

with time-independent probabilities $P_{\sigma}^{s t}$ given by expression (7). We analyze the stability of this solution by linearizing the set of equations in terms of small deviations $\sim \exp (\lambda t)$ from the time-independent solution [Eq. (17)], considering the bias voltage $V_{b} \approx V_{\uparrow}$ and the temperature $k_{B} T \ll \Delta \epsilon$ being low enough to fulfill $f_{\mathrm{F}}^{\sigma}\left(V_{b}\right) \approx 0, f_{\mathrm{N}}^{\downarrow}\left(V_{b}\right) \approx 1$. Thus transforming system [Eq. (16)] into a set of algebraic equations, we obtain the characteristic equation of the fourth order in the Lyapunov exponent $\lambda$, i.e.,

$$
a_{4} \lambda^{4}+a_{3} \lambda^{3}+a_{2} \lambda^{2}+a_{1} \lambda+a_{0}=0
$$

with the coefficients

$$
\begin{gathered}
a_{0}=\Gamma_{\mathrm{N}}^{2} A_{1}, \\
a_{1}=\Gamma_{\mathrm{N}} A_{2}+\mathcal{L} \mathcal{N} e \Gamma_{\mathrm{N}}^{3} A_{3} \vartheta \\
a_{2}=1+\mathcal{L} \mathcal{N} e \Gamma_{\mathrm{N}}^{2} A_{3}\left(\gamma_{\uparrow}+\gamma_{\downarrow}\right)+\mathcal{L} C \Gamma_{\mathrm{N}}^{2} A_{1}, \\
a_{3}=\mathcal{L} \mathcal{N} e \Gamma_{\mathrm{N}} A_{3}+\mathcal{L} C \Gamma_{\mathrm{N}} A_{2}, \\
a_{4}=\mathcal{L} C,
\end{gathered}
$$

where

$$
\begin{gathered}
A_{1} \equiv\left(1+\gamma_{\uparrow}\right)\left(1+\gamma_{\downarrow}\right)-f_{\mathrm{N} \uparrow}, \\
A_{2} \equiv 2+\gamma_{\uparrow}+\gamma_{\downarrow}, \\
A_{3} \equiv \frac{1}{2} f_{\mathrm{N} \uparrow}^{\prime} \frac{\gamma_{\downarrow}\left(1+\gamma_{\uparrow}\right)}{\left(1+\gamma_{\uparrow}\right)\left(1+\gamma_{\downarrow}\right)-f_{\mathrm{N} \uparrow}} .
\end{gathered}
$$

The analysis of the characteristic polynomial in the complex $\lambda$ plane, namely, counting the winding number of its phase as the variable encircles the $\operatorname{Re}(\lambda)>0$ half plane, shows that two roots of Eq. (18) are always real and negative while the other two have the real part changing its sign as $\mathcal{L}$ passes through a critical value $\mathcal{L}_{c}$. In order to find the critical values of the inductance at which the real part of two complex conjugated Lyapunov exponents is equal to 0 , we insert 
$\lambda=i \omega$ (where $\omega$ is real) into the characteristic [Eq. (18)] and obtain a set of two equations

$$
\begin{gathered}
a_{4}(\mathcal{L}) \omega^{4}-a_{2}(\mathcal{L}) \omega^{2}+a_{0}=0, \\
a_{3}(\mathcal{L}) \omega^{2}-a_{1}(\mathcal{L})=0
\end{gathered}
$$

from which the critical inductance $\mathcal{L}_{c}$ and the corresponding roots $\lambda_{1,2}= \pm i \omega_{c}$ of the characteristic equation are found.

When the tunneling rates, which control the average electron populations of the dots, are the fastest rates in the system, NDC leads to the well-known electrodynamical instability of the time-independent current flow. It is interesting to note that in the system under consideration an instability of the time-independent regime arises even in the case when the RC time $t_{R C} \equiv\left|R_{d}\right| C$, where the differential resistance $R_{d}$ $\equiv\left(\mathcal{N} d j_{0} / d V_{b}\right)^{-1}$ is defined by Eq. (9), is the shortest time scale in the system,

$$
t_{R C} \ll \Gamma_{\mathrm{N}}^{-1}, \quad t_{R C} \ll \sqrt{\mathcal{L} C} .
$$

Taking into account realistic values of the junction parameters $l \sim 10 \mathrm{~nm}, d \sim 1 \mathrm{~nm}, \varepsilon \sim 10^{-11} \mathrm{~F} / \mathrm{m}$, and the operating temperature $T \sim 50 \mathrm{mK}$, one finds that the inequalities [Eq. (22)] are well fulfilled down to $\gamma_{\sigma} \gtrsim 10^{-2}$ and inductances $\mathcal{L} \mathcal{N} \gamma_{\sigma}^{4} \gg 10^{-10} H$.

Conditions [Eq. (22)] permit skipping of all terms containing $C$ in system [Eq. (16)] and coefficients [Eq. (19)], i.e., putting there $C=0$. In this case the set of Eq. (21) reduces to an algebraic quadratic equation for $\mathcal{L}$ with roots

$$
\mathcal{L}^{( \pm)}=\frac{R_{d}}{\Gamma_{\mathrm{N}}} \frac{\left[\varphi \pm \sqrt{\varphi^{2}-4\left[\left(1+\gamma_{+}\right)^{2}-1\right] \vartheta}\right]}{\left[\left(1+\gamma_{\uparrow}\right)\left(1+\gamma_{\downarrow}\right)-f_{\mathrm{N} \uparrow}\right] \gamma_{+}},
$$

where $\vartheta\left(\gamma_{\uparrow}, \gamma_{\downarrow}\right)$ is defined by Eq. (10); $\gamma_{+} \equiv \gamma_{\uparrow}+\gamma_{\downarrow}$ and $\varphi\left(\gamma_{\uparrow}, \gamma_{\downarrow}, V_{b}\right) \equiv 1-f_{\mathrm{N} \uparrow}-2 \gamma_{\uparrow}-\gamma_{+}^{2}$.

As it follows from Eq. (23), in the case of $R_{d}<0$ (and hence $\vartheta<0$ ), one root is negative and the other one is positive. Therefore, there is only one critical value of the inductance $\mathcal{L}_{c}=\mathcal{L}^{(-)}$at which the system looses its stability. On the other hand, easily seen from coefficients [Eq. (19)], in the absence of external inductance $(\mathcal{L}=0)$ the characteristic $[\mathrm{Eq}$. (18)] reduces to the second order with both solutions having real parts $\operatorname{Re}(\lambda)<0$, indicating that the fixed point is stable for any choice of the other parameters. From there it follows that in the range of parameters in which the differential resistance $R_{d}$ is negative (light gray area in Fig. 6), the timeindependent solution [Eq. (17)] is stable if $0 \leq \mathcal{L} \leq \mathcal{L}_{c}$ and, if the inductance exceeds the critical value $\mathcal{L}>\mathcal{L}_{c}$, the system looses its stability.

Following from Eq. (23), the presented system reveals one peculiar property: it may also loose its stability in the case of a positive differential resistance $R_{d} \geq 0$ (that is, $\vartheta$ $>0$ ). In the range of $\gamma_{\sigma}$ parameters satisfying

$$
\vartheta \geq 0 ; \quad \varphi^{2} \geq 4\left[\left(1+\gamma_{\uparrow}+\gamma_{\downarrow}\right)^{2}-1\right] \vartheta
$$

(shown as a dark gray area in Fig. 6) both roots $\mathcal{L}^{( \pm)}$are real and positive and hence there are two critical values of the inductance $\mathcal{L}_{c}=\mathcal{L}^{(-)}$and $\mathcal{L}_{c}^{\diamond}=\mathcal{L}^{(+)}$. One can find that the

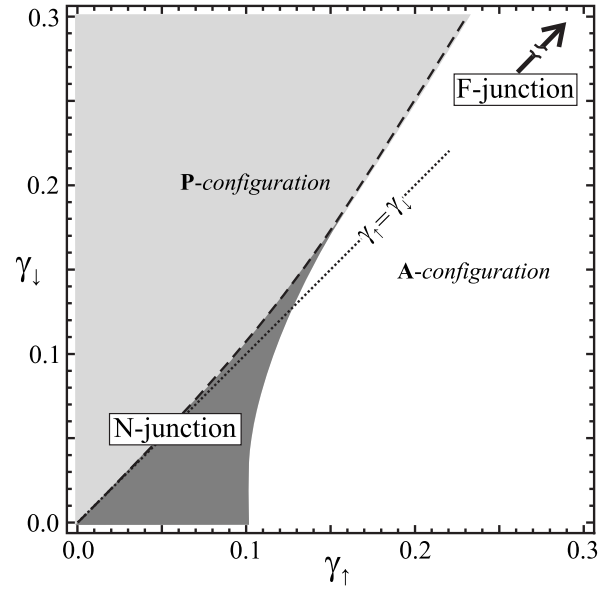

FIG. 6. The instability condition in $\left(\gamma_{\uparrow}, \gamma_{\downarrow}\right)$-parameter space: the light gray area shows the range of parameters in which $R_{d}<0$ and the instability arises for $\mathcal{L}>\mathcal{L}_{c}$. The dark gray area shows the range of parameters in which $R_{d}>0$ and the instability arises for $\mathcal{L}_{c}<\mathcal{L}$ $<\mathcal{L}_{c}^{\diamond}$. Here $V_{b}=V_{\uparrow}$ and $k_{B} T / \Delta \epsilon=0.05$.

system is unstable for inductance laying in the interval $\mathcal{L}_{c}$ $<\mathcal{L}<\mathcal{L}_{c}^{\diamond}$ and stable otherwise.

All the features mentioned above are most transparent in the limit of a highly asymmetric $\mathrm{N}$ junction $\gamma_{\sigma} \ll 1$. In this case the critical values of the inductances $\mathcal{L}_{c}$ and $\mathcal{L}_{c}^{\diamond}$, as well as the corresponding frequencies $\omega_{c}=\omega\left(\mathcal{L}_{c}\right)$ and $\omega_{c}^{\vartheta}=\omega\left(\mathcal{L}_{c}^{\diamond}\right)$, are

$$
\begin{gathered}
\mathcal{L}_{c} \approx \frac{4}{e \Gamma_{\mathrm{N}}^{2} \mathcal{N} f_{\mathrm{N} \uparrow}^{\prime} \gamma_{\downarrow}} ; \quad \omega_{c} \approx \Gamma_{\mathrm{N}} \sqrt{1-f_{\mathrm{N} \uparrow}}, \\
\mathcal{L}_{c}^{\diamond} \approx \frac{2}{e \Gamma_{\mathrm{N}}^{2} \mathcal{N} f_{\mathrm{N} \uparrow}^{\prime}} \frac{\left(1-f_{\mathrm{N} \uparrow}\right)^{2}}{\gamma_{\downarrow}\left(\gamma_{\uparrow}+\gamma_{\downarrow}\right) \vartheta} ; \quad \omega_{c}^{\diamond} \approx \Gamma_{\mathrm{N}} \sqrt{\vartheta} .
\end{gathered}
$$

From expression (25) it is clearly seen that in the limit $\gamma_{\sigma} \ll 1$ the first critical value $\left(\mathcal{L}_{c} ; \omega_{c}\right)$ does not depend on sign of differential resistance while the second one $\left(\mathcal{L}_{c}^{\diamond} ; \omega_{c}^{\diamond}\right)$ appear as soon as IVC attains positive differential conductance $(\vartheta>0)$. Therefore, the instability takes place both for $R_{d}<0$ and $R_{d}>0$ as soon as the inductance exceeds $\mathcal{L}_{c}$, but, as mentioned before, stability is established again as soon as $\mathcal{L} \geq \mathcal{L}_{c}^{\diamond}$ if the differential resistance is positive.

The above-mentioned instability is a Hopf bifurcation, resulting in the onset of spontaneous, nonlinear, periodic in time, self-excited oscillations of current $J(t)$, voltage drop $V(t)$, average charge $q(t)=e \mathcal{N} P_{c}(t)$, and average spin $s(t)$ $=(1 / 2) \mathcal{N} P_{s}(t)$ in the layer of dots. In the case $\left(\mathcal{L}-\mathcal{L}_{c}\right) / \mathcal{L}_{c}$ $\ll 1$ the frequency of the oscillations $\approx \omega_{c}$. Analytical estimations and numerical calculations show that the critical inductance increases and the oscillations fade out with an increase in the temperature, disappearing at $k_{B} T \sim \Delta \epsilon$. The numerical solutions of system [Eq. (16)] with $C=0$, for an $\mathrm{N}$-type junction in $\mathbf{P}$ configuration with the NDC, in circuit with inductance chosen slightly beyond the critical value, show charge/ spin accumulation, as well as current, orbiting in time along the limit cycle as presented in Fig. 7. An example of limit 
(a)

(b)
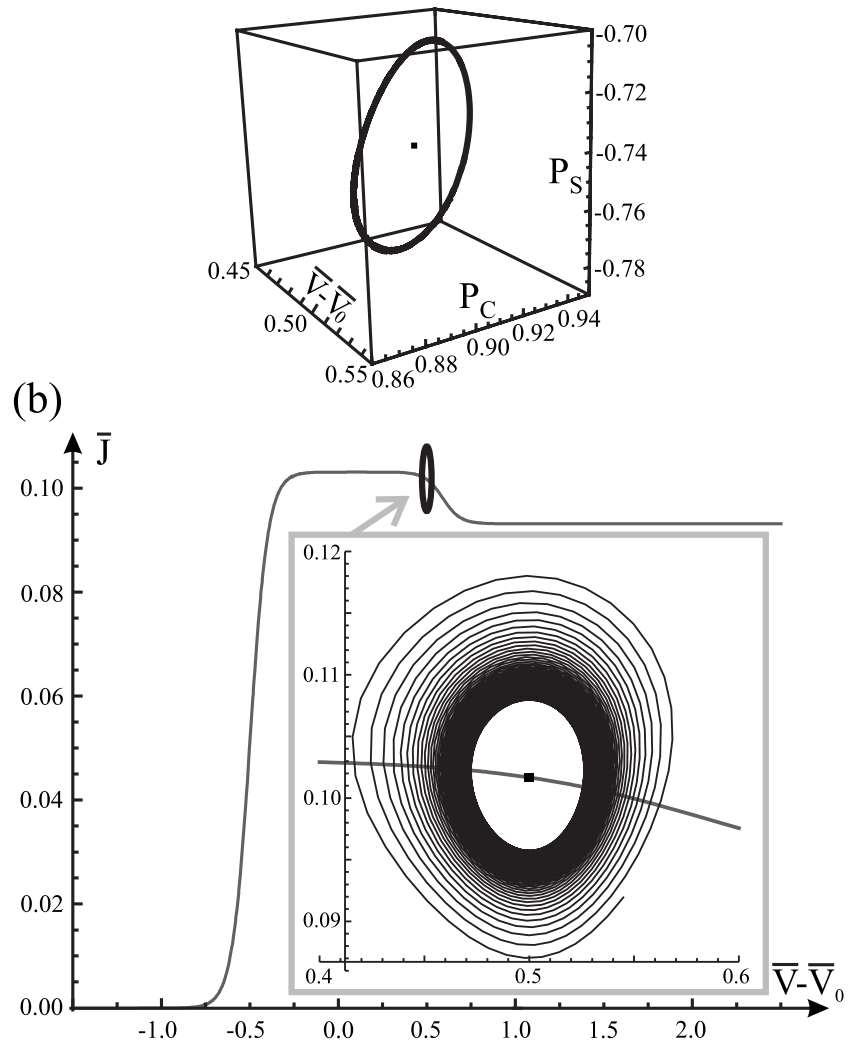

FIG. 7. (a) The limit cycle in $\left(\bar{V}, P_{c}, P_{s}\right)$ space around the fixed point $(0.5,0.905,-0.743)$. (b) The limit cycle in the $(\bar{V}, \bar{J})$ space around the fixed point $(0.5,0.102)$ at the IVC (gray curve). We use scales: $\bar{V}=e V / \Delta \epsilon, \bar{J}=J / e \Gamma_{\mathrm{N}} \mathcal{N}, \overline{\mathcal{L}}=\mathcal{L} e^{2} \Gamma_{\mathrm{N}}^{2} \mathcal{N} / \Delta \epsilon$, and $\bar{\omega}=\omega / \Gamma_{\mathrm{N}}$. Voltage is measured with respect to $\bar{V}_{0}=\left(\bar{V}_{\uparrow}+\bar{V}_{\downarrow}\right) / 2$. Here the choice of parameters is $\bar{V}_{b}=\bar{V}_{\uparrow}, k_{B} T / \Delta \epsilon=0.05, \quad \gamma_{\uparrow}=0.085, \quad \gamma_{\downarrow}$ $=0.115, \quad \xi=0.1, \quad \overline{\mathcal{L}}=8.0$ with critical values for instability $\overline{\mathcal{L}}_{c}$ $=7.8712$, and $\bar{\omega}_{c}=0.5457$. This choice of parameters (P configuration) leads to an IVC with NDC and the frequency of stable oscillations $\bar{\omega}=0.5456$.

cycle for A configuration with positive differential conductance is shown in Fig. 8. The results show critical inductances on the order of $1 \mathrm{mH} / \mathcal{N}$ and critical frequencies on the order of $\Gamma_{\mathrm{N}}$, i.e., $1 \mathrm{GHz}$, independent of $\mathcal{N}$. Typical junction capacitance per dot $C_{1 \mathrm{QD}} \sim 10^{-18} \mathrm{~F}$ introduces just small correction to critical inductance lowering it by $\sim 0.2 \%$ while critical frequency remains unchanged.

\section{CONCLUSION}

We considered the effect of Coulomb blockade correlations on the spin-dependent electronic transport across a layer of quantum dots connecting a normal and a magnetic lead. It was shown that in such a system, under the voltage biasing with an inductor added in series with the junction, an instability in the steady (time-independent) flows of charge and spin may arise. This instability develops into a new stable regime in which the average spin and charge accumulated in the dots oscillate periodically in time. The typical (a)

(b)
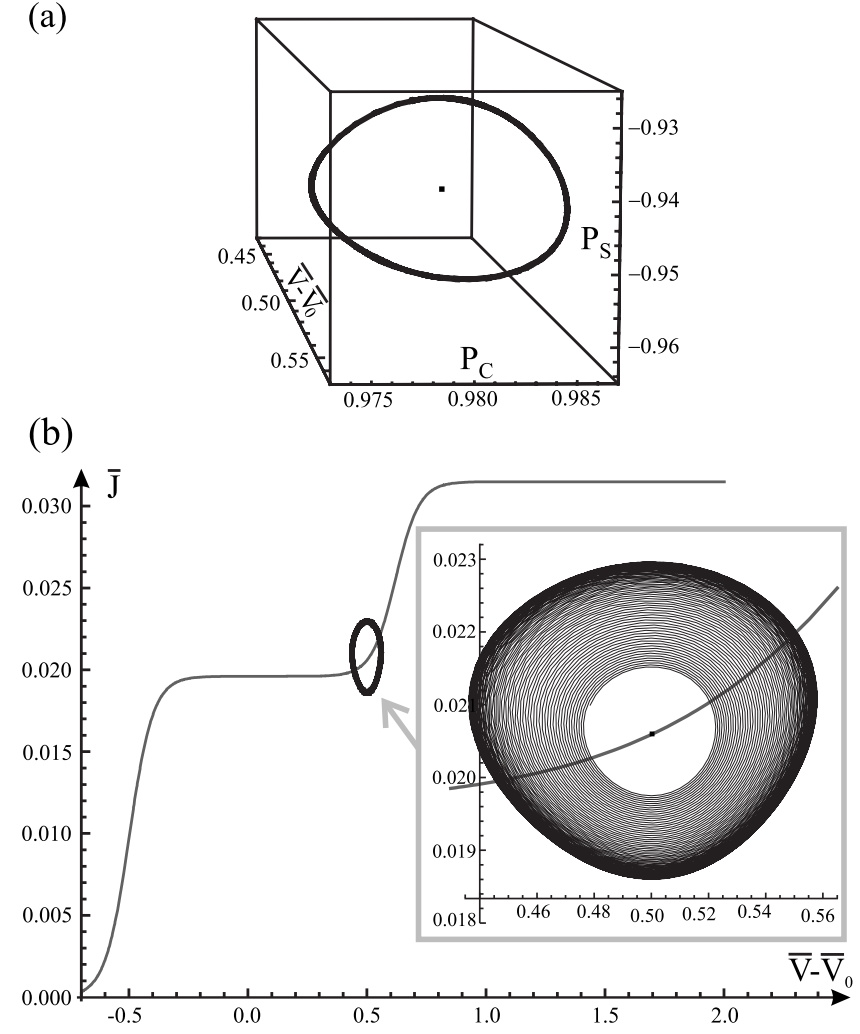

FIG. 8. The limit cycle appearing as the result of the instability in the system with positive differential conductance at the stationary IVC (gray curve) in A configuration, (a) around the stationary points $(0.5,0.981,-0.948)$ in $\left(\bar{V}, P_{c}, P_{s}\right)$ space, and (b) around stationary point $(0.5,0.0206)$ in $(\bar{V}, \bar{J})$ space. Used scales and voltage origin are the same as in Fig. 7. The choice of parameters is $\bar{V}_{b}$ $=\bar{V}_{\uparrow}, k_{B} T / \Delta \epsilon=0.05, \gamma_{\uparrow}=0.08, \gamma_{\downarrow}=0.02$, and $\overline{\mathcal{L}}=45.0$. The critical values between which the instability takes place are $\overline{\mathcal{L}}_{c}=41.112$; $\bar{\omega}_{c}=0.5883$ and $\overline{\mathcal{L}}_{c}^{\diamond}=257.301 ; \bar{\omega}_{c}^{\diamond}=0.3272$. The frequency of the stable oscillations $\bar{\omega}=0.5865$.

frequency of the oscillations is on the order of $1 \mathrm{GHz}$ for realistic junction parameters. In contrast to the standard electric instability of an RLC circuit with a negative differential resistance, in the system under consideration spin accumulation in the dots results in an instability which occurs even in the case of an RL circuit with a positive differential resistance.

\section{ACKNOWLEDGMENTS}

Financial support from the Swedish VR and SSF, the European Commission (FP7-ICT-2007-C; Project No. 225955 STELE) and the Korean WCU program funded by MEST/ NFR (Grant No. R31-2008-000-10057-0) is gratefully acknowledged.

\section{APPENDIX}

The rate equation 


$$
\frac{d}{d t}\left(\begin{array}{c}
P_{0} \\
P_{\uparrow} \\
P_{\downarrow}
\end{array}\right)=\sum_{i=\mathrm{N}, \mathrm{F}}\left(\begin{array}{ccc}
-\left(\Gamma_{i}^{\uparrow, L \rightarrow \mathrm{QD}}+\Gamma_{i}^{\downarrow, L \rightarrow \mathrm{QD}}\right) & \Gamma_{i}^{\uparrow, \mathrm{QD} \rightarrow L} & \Gamma_{i}^{\downarrow, \mathrm{QD} \rightarrow L} \\
\Gamma_{i}^{\uparrow, L \rightarrow \mathrm{QD}} & -\Gamma_{i}^{\uparrow, \mathrm{QD} \rightarrow L} & 0 \\
\Gamma_{i}^{\downarrow, L \rightarrow \mathrm{QD}} & 0 & -\Gamma_{i}^{\downarrow, \mathrm{QD} \rightarrow L}
\end{array}\right)\left(\begin{array}{c}
P_{0} \\
P_{\uparrow} \\
P_{\downarrow}
\end{array}\right) .
$$

where eight $\Gamma$ 's are the rates of electron tunneling between the lead (L) and the dot (QD), calculated using Fermi golden rule and written in compact form as $\Gamma_{i}^{\sigma, \eta}\left(V_{i}\right)$ $=\frac{2 \pi}{\hbar}\left|\tau_{i}\right|^{2} \int f^{\eta}\left[E_{i, \sigma}(\vec{p})+e V_{i}-\mu_{i}\right] \delta\left[E_{i, \sigma}(\vec{p})+e V_{i}-\epsilon_{\sigma}\right] d \vec{p}$, resulting in the expression

$$
\begin{aligned}
\Gamma_{i}^{\sigma, \eta}(V)= & \frac{2 \pi}{\hbar}\left|\tau_{i}\right|^{2} f^{\eta}\left(\epsilon_{\sigma}+\varsigma_{i} e V-\mu_{0}\right) \\
& \times g_{i}\left[\varepsilon_{i}+\epsilon_{\sigma}-\frac{1}{2}\left(1+\varsigma_{i}\right) \sigma I+\varsigma_{i} e V\right] .
\end{aligned}
$$

Here index $i=\mathrm{N}, \mathrm{F}$ denotes process between $\mathrm{QD}$ and $\mathrm{N} / \mathrm{F}$ lead, $\sigma= \pm 1$ stands for spin $\uparrow / \downarrow, \varsigma_{i}=\mp 1$ for $i=\mathrm{N} / \mathrm{F}$, while $\eta=L \rightarrow \mathrm{QD}, \mathrm{QD} \rightarrow L$ denotes the tunneling process from the lead to the QD and vice versa, respectively. $\tau_{i}$ is averaged, energy-independent matrix element of tunneling Hamiltonian (2) between QD and the lead $i, g_{i}(\varepsilon)$ is electron density of states in lead $i$ at given energy $\varepsilon$, while $f^{\eta}(\varepsilon)$ denotes Fermi function $f(\varepsilon)$ for $L \rightarrow \mathrm{QD}$ process, and $1-f(\varepsilon)$ for $\mathrm{QD} \rightarrow L$ process respectively. Since $g(V)$ is slowly varying function along the voltage interval $\left(V_{\downarrow}, V_{\uparrow}\right)$, we approximate the tunneling rates as

$$
\Gamma_{i}^{\sigma, \eta}(V) \approx f^{\eta}\left(\epsilon_{\sigma}+\varsigma_{i} e V-\mu_{0}\right) \Gamma_{i}^{\sigma},
$$

where $\Gamma_{i}^{\sigma}=(2 \pi / \hbar)\left|\tau_{i}\right|^{2} g_{i}^{\sigma}$ is the voltage-independent "bare" tunneling rate, by approximating $g_{i}^{\sigma} \approx g\left[\varepsilon_{i}-\frac{1}{2}\left(1+\varsigma_{i}\right) \sigma I\right]$ for $e V$ and $\epsilon_{\sigma}-\mu_{0} \ll \varepsilon_{i}, I\left(\epsilon_{\sigma}-\mu_{0}\right.$ can be adjusted small using the gate voltage).
${ }^{1}$ K. Ono, D. G. Austing, Y. Tokura, and S. Tarucha, Science 297, 1313 (2002).

${ }^{2}$ P. Recher, E. V. Sukhorukov, and D. Loss, Phys. Rev. Lett. 85, 1962 (2000).

${ }^{3}$ A. A. Shokri, M. Mardaani, and K. Esfarjani, Physica E (Amsterdam) 27, 325 (2005).

${ }^{4}$ A. Brataas, Y. V. Nazarov, J. Inoue, and G. E. W. Bauer, Phys. Rev. B 59, 93 (1999).

${ }^{5}$ H. Imamura, S. Takahashi, and S. Maekawa, Phys. Rev. B 59, 6017 (1999).

${ }^{6}$ W. Rudziński and J. Barnaś, Phys. Rev. B 64, 085318 (2001).

${ }^{7}$ L. Y. Gorelik, S. I. Kulinich, R. I. Shekhter, M. Jonson, and V. M. Vinokur, Phys. Rev. Lett. 95, 116806 (2005).

${ }^{8}$ J. S. Helman and B. Abeles, Phys. Rev. Lett. 37, 1429 (1976).

${ }^{9}$ L. F. Schelp, A. Fert, F. Fettar, P. Holody, S. F. Lee, J. L. Maurice, F. Petroff, and A. Vaurès, Phys. Rev. B 56, R5747 (1997).

${ }^{10}$ S. Takahashi and S. Maekawa, Phys. Rev. Lett. 80, 1758 (1998).

${ }^{11}$ J. Barnaś and A. Fert, Phys. Rev. Lett. 80, 1058 (1998).

${ }^{12}$ L. Y. Gorelik, S. I. Kulinich, R. I. Shekhter, M. Jonson, and V.
M. Vinokur, Appl. Phys. Lett. 90, 192105 (2007).

${ }^{13}$ J. R. Hauptmann, J. Paaske, and P. E. Lindelof, Nat. Phys. 4, 373 (2008).

${ }^{14}$ J. Weis, R. J. Haug, K. v. Klitzing, and K. Ploog, Phys. Rev. Lett. 71, 4019 (1993).

${ }^{15}$ M. M. Deshmukh and D. C. Ralph, Phys. Rev. Lett. 89, 266803 (2002).

${ }^{16}$ M. C. Rogge, F. Cavaliere, M. Sassetti, R. J. Haug, and B. Kramer, Physica E (Amsterdam) 32, 442 (2006).

${ }^{17}$ F. Elste and C. Timm, Phys. Rev. B 73, 235305 (2006).

${ }^{18}$ F. M. Souza, J. C. Egues, and A. P. Jauho, Phys. Rev. B 75, 165303 (2007).

${ }^{19}$ J. König, J. Schmid, H. Schoeller, and G. Schon, Phys. Rev. B 54, 16820 (1996); H. Schoeller, in Mesoscopic Electron Transport, edited by L. L. Sohn, L. P. Kouwenhoven, and G. Schön (Kluwer, Dordrecht, 1997); M. Braun, J. König, and J. Martinek, Phys. Rev. B 70, 195345 (2004).

${ }^{20}$ J. König and J. Martinek, Phys. Rev. Lett. 90, 166602 (2003).

${ }^{21}$ L. I. Glazman and K. A. Mateev, JETP Lett. 48, 445 (1988). 